\title{
Supervised Learning Using a Symmetric Bilinear Form for Record Linkage
}

\author{
Daniel Abrila,c,*, Vicenç Torra ${ }^{\mathrm{a}, \mathrm{d}}$, Guillermo Navarro-Arribas ${ }^{\mathrm{b}}$ \\ ${ }^{a}$ IIIA, Institut dInvestigació en Intel.ligència Artificial, CSIC, Consejo Superior de \\ Investigaciones Científicas. Campus UAB s/n, 08193, Bellaterra, Spain \\ ${ }^{b}$ DEIC, Dep. Enginyeria de la Informació $i$ de les Comunicacions, UAB, Universitat \\ Autònoma de Barcelona. Campus UAB s/n, 08193, Bellaterra, Spain \\ ${ }^{c} U A B$, Universitat Autònoma de Barcelona. Campus UAB s/n, 08193, Bellaterra, Spain \\ ${ }^{d}$ School of Informatics, University of Skövde. 54128 Skövde, Sweden
}

\section{Abstract}

Record Linkage is used to link records of two different files corresponding to the same individuals. These algorithms are used for database integration. In data privacy, these algorithms are used to evaluate the disclosure risk of a protected data set by linking records that belong to the same individual. The degree of success when linking the original (unprotected data) with the protected data gives an estimation of the disclosure risk.

In this paper we propose a new parameterized aggregation operator and a supervised learning method for disclosure risk assessment. The parameterized operator is a symmetric bilinear form and the supervised learning method is formalized as an optimization problem. The target of the optimization problem is to find the values of the aggregation parameters that maximize the number of re-identification (or correct links). We evaluate and compare our proposal with other non-parametrized variations of record linkage, such as those using the Mahalanobis distance and the Euclidean distance (one of the most used approaches for this purpose). Additionally, we also compare it with other previously presented parameterized aggregation operators for record linkage such as the weighted mean and the Choquet integral. From these comparisons we show how the proposed aggregation operator is able to overcome or at least achieve similar results than the other

\footnotetext{
* Corresponding author

Email addresses: dabril@iiia.csic.es (Daniel Abril ), vtorra@his.se (Vicenç Torra), guillermo.navarro@uab.cat (Guillermo Navarro-Arribas)
} 
parameterized operators. We also study which are the necessary optimization problem conditions to consider the described aggregation functions as metric functions.

Keywords: record linkage, data privacy, disclosure risk, supervised learning, bilinear form, fuzzy measure, Choquet integral

\section{Introduction}

In this paper we introduce a new variation of the supervised learning approach for record linkage. This consists of a symmetric bilinear form and a supervised learning approach. This aggregation function relies on a symmetric weighting matrix and it can be considered a Mahalanobis-based distance when the weighting matrix is positive semi-definite. We also present a couple of supervised learning approaches adapted to this symmetric bilinear function. Both are different approximations to obtain a semi-definite weighting matrix. Additionally, we study the previously proposed aggregators, the weighted mean and the Choquet integral, and propose different problem formalizations to use them as distances. Finally, we present a comparison in terms of accuracy and time between all disclosure risk approaches. Those are the non-parameterized functions such as the Euclidean distance and the Mahalanobis distance, the literature parameterized aggregators with their corresponding proposed modifications and finally the proposed symmetric bilinear approach.

The outline of this paper is as follows. Section 2 briefly introduces the state-of-the-art and related work. In Section 3, we review some concepts needed in the rest of the paper. Then, in Section 4, we review some standard distances used in record linkage, two parametrized aggregation operators used in previous works and finally the proposed bilinear function. In Section 5, we describe the optimization problem. That is, the supervised learning approach for distance-based record linkage. The evaluation of the method in the context of data privacy is done in Section 6. Finally, Section 7 presents the conclusions of the paper.

\section{Related work}

Record linkage is the process of finding quickly and accurately two or more records distributed in different databases (or data sources in general) 
that make reference to the same entity or individual. This term was initially introduced in the public health area in [1], when files of individual patients were brought together using name, date-of-birth, and some other information. In the following years, this idea was deeply developed in $[2,3,4]$, and nowadays it is a popular technique.

Record linkage is one of the existing preprocessing techniques used for data cleaning [5, 6], and for improving data quality [7]. For example, record linkage can be used to scrutinize databases to improve dirty data removing duplicate records [8], correcting data entry mistakes, transcription errors, and solving problems due to lack of standards for recording data fields, etc.

In addition, it is also a popular technique employed to integrate different data sets that provide information regarding the same entities [9, 10]. For instance, consider the linkability of a census dataset with health records. A step forward in this direction is the merging of very large databases. A clear example of this database integration is the initiative recently launched by the UK government to make all its data available as RDF (Resource Description Framework) with the purpose of enabling data to be linked together [11]. Similar initiatives also have been applied in the USA [12].

In the last years, record linkage techniques have also emerged in the data privacy context. Many government agencies and companies are collecting massive amounts of confidential data, such as medical records, income credit rating or even several types of test scores. In order to perform different kind of studies these datasets are analyzed by their owners or more commonly by third parties, creating a conflict between individual's right to privacy and the society's need to know. So, it is fundamental to provide privacy to databases against disclosure of confidential information. Privacy Preserving Data Mining [13] and Statistical Disclosure Control [14] research on methods and tools for ensuring the privacy of these data. The idea behind all of these developed methods is to modify statistical data, also called microdata, so that they can be published without giving away confidential information that can be linked to specific respondents and also achieve it with minimum loss of detail. Record linkage permits the evaluation of the disclosure risk of protected data $[15,16]$. In this context record linkage could be applied by an attacker, who tries to link his own information (original) with the protected one to obtain some new and unknown information. If the links can be established, the attacker can re-identify individuals from the protected data, and the protection is said to be broken. This is also applicable to model the worst-case scenario, where the attack attempts to link all records from the 
original data (the most comprehensive information an attacker can use) to the protected data. This gives an estimation of the chances that an attacker will be able to re-identify records in the protected data. The estimation is usually used as a disclosure risk measure of the protection method applied to protect the data. That is, the percentage of correctly linked records between the protected dataset and the original dataset is taken as a measure for the disclosure risk of the data. Thus, the higher the percentage of correctly linked records, the higher the risk of disclosure. This approach to measure the disclosure risk of protected data was initially introduced in [17] and adopted in much of the subsequent literature such as $[18,16,15]$. Note also that sampling is not taken into account in this approach, which means assuming that the intruder knows the sampled individuals in the data set. This is a common practice in the previously cited works.

In addition, Domingo-Ferrer and Torra [18] defined a general score to qualitatively rank protection methods. This score is the combination of disclosure risk techniques, to evaluate the risk of re-identification, and other techniques, which readily quantifies the information loss of a protected data set using analytical measures (either generic or data-use-specific).

We introduce a new optimization problem for distance-based record linkage and its application to data privacy. The performance of this approach depends critically on a given distance. The choice of a distance over an input space always has been a key issue in many machine learning algorithms. Due to the problems of the commonly used Euclidean distance, which assumes that each feature is equally important and independent from the others, distance metric learning has emerged as a research topic [19]. Although the origins of metric learning can be traced in earlier works, Xing et al. [20] were pioneers within this research area. Similar to our proposal, they parameterize the Euclidean distance using a symmetric positive semi-definite matrix $\Sigma \succeq 0$ to ensure the non-negativity of the metric. Their algorithm maximizes the sum of distances between dissimilar points, while keeping closer the set of distances between similar points. However, despite its simplicity, the method is scalable, because it has to perform many eigenvalue decompositions. [21] proposed a method for learning distance metrics from relative comparisons such as $a$ is closer to $b$ than $a$ is to $c$. This relies on a less general Mahalanobis distance learning in which for a given matrix $a$, only a diagonal matrix $W$ is learnt such that $\Sigma=A^{\prime} W A$. More recently, [22] proposed a framework for learning the weighted Euclidean subspace based on pairwise constrains and cluster validity, where the best clustering can be achieved. Beliakov et al. 
[23] considered the problem of metric learning in semi-supervised clustering defining the Choquet integral with respect to a fuzzy measure as an aggregation distance. The authors investigate necessary and sufficient conditions for the discrete Choquet integral to define a metric. Weinberger et al. [24] proposed a new classification algorithm, the Large Margin $k$-nearest neighbour $(\mathrm{LMNN})$, in which is learned a Mahalanobis distance. This metric is trained with the goal that the $k$-nearest neighbors always belong to the same class while examples from different classes are separated by a large margin. However, Sun and Chen [25] show that LMNN cannot satisfactorily represent the local metrics which are respectively optimal in different regions of the input space and they propose a local distance metric learning method, a hierarchical distance metric learning for LMNN, which first groups data points in a hierarchical structure and then learns the distance metric for each hierarchy. The authors use a classification algorithm, Large Margin Nearest Neighbor (LMNN) to classify points in the hierarchical structure. The paper concludes that hierarchical distance works well when the number of classes is large but that it does not improve the results of LMNN when the number of classes is small.

One of the most important challenges associated with supervised metric learning approaches, specially in Mahalanobis-based distances is the satisfaction of the positive semi-definiteness. In the literature there are different approximations, from several matrix simplifications to modern semi-definite programming methods within the operations research field. Some $\Sigma$ simplifications force it to be diagonal and so $\Sigma$ is positive semi-definite if and only if all diagonal entries are non-negative. This simplification reduces the number of parameters drastically and makes the optimization problem a linear program. Higham [26] proposed an algorithm to find the nearest correlation matrix, symmetric positive semi-definite matrix with unit diagonal, to a given symmetric matrix by means of a projection from the symmetric matrices onto the correlation matrices, with respect to a weighted Frobenius form. Semi-definite programming (SDP), is a kind of convex programming which evolved from linear programming. While, a linear programming problem is defined as the problem of maximizing or minimizing a linear function subject to a set of linear constraints, semidefinite programming is defined as the problem of maximizing or minimizing a linear function subject to a set of linear constraints and a "semi-definite" constraint, a special form of non-linear constraints. Therefore, the semi-definite constraint is what differentiates SDPs from LPs. 
Motivated by this research line some researchers started to work on supervised metric learning approaches for disclosure risk assessment. These methods rely on distance-based record linkage techniques and so, by means of introducing must-link and cannot-link constraints and an aggregator operator they are able to formalize an optimization problem. Thus, by using global optimization mechanisms they are able to solve the optimization problem and get the aggregator parameters that maximize the number of correct re-identification between records (individuals) from two datasets. This research continues the line started by Torra et al. [27] where two different optimization problems were presented: one relies on a weighted arithmetic mean and other on the Ordered Weighted Aggregation (OWA) operator. Later on, Abril et al. [28] improved the previous weighted mean supervised learning approach and compared it with the current distance-based record linkage technique. They also made an extensive disclosure risk comparison between a large set of well-known protection methods. Besides, they show the relevance of knowing the aggregation parameters for protection practitioners. Afterwards, Abril et al. [29] proposed a similar supervised metric learning approach, but this time using a fuzzy integral. That is, the Choquet integral as an aggregation operator, which permits the integration of a function with respect to a fuzzy measure. They improved the re-identification accuracies achieved by standard distance-based record linkage methods as well as by the previous presented approaches. Moreover, thanks to fuzzy measures they could extract much more information about the linkage process than using the weighted mean. However, using the Choquet integral makes the optimization problem much more complex, specially for the number of problem constraints and the number of parameters to be found.

\section{Preliminaries}

In this section we review some ideas and definitions that are needed to follow the rest of the paper. We explain the notation we use as well as how the record linkage is applied in the data privacy area.

\subsection{Record Linkage}

As stated in the introduction, record linkage is a re-identification method that links records in one file with records in another file that correspond to the same individuals. There are two extensively used approaches of record linkage. 
- Distance based record linkage (DBRL). This approach [30] links each record $a$ of a file $X$ to the closest record $b$ in a file $X^{\prime}$. The closest record is defined in terms of a distance function.

- Probabilistic record linkage (PRL). In this case, the matching algorithm uses the linear sum assignment model to choose which pairs of records must be matched. In order to compute this model, the EM (Expectation - Maximization) algorithm [31,32] is normally used. Informally, we consider records $a$ and $b$ of files $X$ and $X^{\prime}$, respectively, represented in terms of a set of variables $V 1, \ldots, V_{n}$. That is, $a=\left(V_{1}(a), \ldots, V_{n}(a)\right)$ and $b=\left(V_{1}(b), \ldots, V_{n}(b)\right)$. Then, we define a coincidence vector $\gamma(a, b)=\left(\gamma_{1}(a, b) \ldots \gamma_{n}(a, b)\right)$, where $\gamma_{i}(a, b)$ is defined as 1 if $V_{i}(a)=V_{i}(b)$ and as 0 if $V_{i}(a) \neq V_{i}(b)$. According to some criterion defined over these coincidence vectors, pairs are classified as linked pairs (LP) or non-linked pairs (NP). This concrete method was introduced in [33], although probabilistic record linkage was first presented in [4].

The work in this paper is focused on distance based record linkage, which is further described in Section 4.

\subsection{Record linkage in data privacy}

Record linkage is a common approach to disclosure risk assessment in data privacy. It is used to model how an attacker links his information with a published (protected) data set. We give a summary of this problem below. See e.g., $[16,28]$ for details.

A dataset $X$ can be viewed as a matrix with $N$ rows (records) and $V$ columns (variables), where each row refers to a single individual. The variables in a dataset can be classified in two different categories:

- Identifiers: variables that can identify an individual unambiguously, e.g., the passport number. Let $X_{i d}$ denote these variables in $X$.

- Quasi-identifiers: variables that are not able to identify a single individual when they are used alone, but that can unequivocally identify an individual when combining several of them. Among the quasi-identifier variables, we distinguish between confidential $\left(X_{c}\right)$ and non-confidential $\left(X_{n c}\right)$, depending on the kind of information they contain. For example, the zip code is a non-confidential quasi-identifier and the salary is an example of a confidential quasi-identifier. 
To avoid disclosure, when we want to publish a data set $X$, where $X=$ $X_{i d}\left\|X_{n c}\right\| X_{c}$, a protection method should be applied to $X$, leading to a protected data set $X^{\prime}=\rho(X)$. This protection process is usually as follows; first, a protection method $\rho$ is used to protect the non-confidential quasiidentifiers, i.e., $X_{n c}^{\prime}=\rho\left(X_{n c}\right)$. Second, to ensure the privacy of the individuals the identifiers are either removed or encrypted and, their confidential quasi-identifiers are not modified because they are the data of interest for third parties. Therefore, the protected data set consists of $X^{\prime}=X_{n c}^{\prime} \| X_{c}$.

In order to evaluate the disclosure risk of releasing $\rho(X)$, we model the behaviour of an attacker applying record linkage to the pair $(X, \rho(X))$. The more records are re-identified, the larger the disclosure risk. This scenario, which was first used in [18] to compare several protection methods, has been adopted in other works like [15].

\section{Distance-Based Record Linkage}

The main point in distance-based record linkage is the definition of the distance function used to match the records. Different distances can be found in the literature, each obtaining different results. In this section we start reviewing two of the most frequently used distances on record linkage, the Euclidean and the Mahalanobis distances. Then, we introduce the parametrized distances, which we will use together with a supervised learning process to obtain the parameters yielding the highest number of re-identifications. Examples of these parametrized distances are those based on the weighted mean and the Choquet integral. In this vein we introduce a parametrized symmetric bilinear function in which the parameters are represented by a weighting matrix.

We adopt the definition of distance function and metric from [34], where a distance function is defined in a less restrictive way than a metric.

Definition 1. Let $X$ be a set. A function $d: X \times X \rightarrow \mathbb{R}$ is called a distance (or dissimilarity) on $X$ if, for all $a, b, \in X$, there holds:

1. $d(a, b) \geq 0$ (non-negativity)

2. $d(a, a)=0$ (reflexivity)

3. $d(a, b)=d(b, a)$ (symmetry)

Definition 2. Let $X$ be a set. A function $d: X \times X \rightarrow \mathbb{R}$ is called a metric on $X$ if, for all $a, b, c \in X$, there holds: 
1. $d(a, b) \geq 0$ (non-negativity)

2. $d(a, b)=0$ iff $a=b$ (identity of indiscernibles)

3. $d(a, b)=d(b, a)$ (symmetry)

4. $d(a, b) \leq d(a, c)+d(c, b)$ (triangle inequality)

Note that other works may consider the terms metric and distance function as the same concept (Definition 2). Then, those works are using terms such as pseudo-metric or pre-metric in order to denote Definition 1.

Now that we have reviewed the properties required by a metric and a distance function, we are going to survey some metrics used in record linkage.

We will use $V_{1}^{X}, \ldots, V_{n}^{X}$ and $V_{1}^{Y}, \ldots, V_{n}^{Y}$ to denote the set of variables of file $X$ and $Y$, respectively. Using this notation, we express the values of each variable of a record $a$ in $X$ as $a=\left(V_{1}^{X}(a), \ldots, V_{n}^{X}(a)\right)$ and of a record $b$ in $Y$ as $b=\left(V_{1}^{Y}(b), \ldots, V_{n}^{Y}(b)\right) . \overline{V_{i}^{X}}$ corresponds to the mean of the values of variable $V_{i}^{X}$.

Definition 3. Given two datasets $X$ and $Y$, the square of the Euclidean distance between two records $a \in X$ and $b \in Y$ for variable-standardized data is defined by:

$$
d^{2} \operatorname{ED}(a, b)=\sum_{i=1}^{n}\left(\frac{V_{i}^{X}(a)-\overline{V_{i}^{X}}}{\sigma\left(V_{i}^{X}\right)}-\frac{V_{i}^{Y}(b)-\overline{V_{i}^{Y}}}{\sigma\left(V_{i}^{Y}\right)}\right)^{2}
$$

where $\sigma\left(V_{i}^{X}\right)$ and $\overline{V_{i}^{X}}$ are the standard deviation and the mean of all the values of variable $V_{i}$ in the dataset $X$, respectively.

It is well known that in the Euclidean distance all the variables contribute equally to the computation of the distance. Because of that all points with the same Euclidean distance to the origin define a sphere. There are other metrics were this property does not hold. For example, the Mahalanobis distance [35] allows us to calculate distances taking into account a different variable contribution by means of weighting these variables. These weights are obtained from the covariances between data variables. Because of this rescaling, points at the same Mahalanobis distance define an ellipse around the mean of the set of variables.

Definition 4. Given two datasets $X$ and $Y$, the square of the Mahalanobis distance between two records $a \in X$ and $b \in Y$ is defined by:

$$
d^{2} M D_{\Sigma}(a, b)=(a-b)^{\prime} \Sigma^{-1}(a-b)
$$


where $(a-b)^{\prime}$ is the transpose of $(a-b)$ and $\Sigma$ is the covariance matrix, computed by $\left[\operatorname{Var}\left(V^{X}\right)+\operatorname{Var}\left(V^{Y}\right)-2 \operatorname{Cov}\left(V^{X}, V^{Y}\right)\right]$, where $\operatorname{Var}\left(V^{X}\right)$ is the variance of variables $V^{X}, \operatorname{Var}\left(V^{Y}\right)$ is the variance of variables $V^{Y}$ and $\operatorname{Cov}\left(V^{X}, V^{Y}\right)$ is the covariance between variables $V^{X}$ and $V^{Y}$.

Any covariance matrix is a symmetric positive semi-definite ${ }^{1}$ matrix, so $d^{2} M D$ satisfies the first metric requirement (Definition 2), because all covariance matrices are always positive semi-definite and it is known that the inverse of a positive definite matrix is always positive definite too. That is, for any vector $v$ and a $n \times n$ positive definite matrix $\Sigma$ the following inequality $v^{\prime} \Sigma^{-1} v>0$ is always satisfied.

Notice that from this definition it follows that when the covariance matrix is the identity matrix, the Mahalanobis distance is reduced to the Euclidean distance.

Let us now focus on the parametrized distances. We first introduce a generic definition of a distance based on aggregation operators [36] and then consider two particularizations of this generic distance.

The generic distance is based on the fact that the Euclidean distance has the same results when it is multiplied it by a constant. Then, we express the Euclidean distance $\left(d^{2} E D(a, b)\right)$ given in Definition 3 as a weighted mean of the distances for the variables. For the sake of simplicity we consider the square of the distances although it is clear that it is not a distance itself, because it does not satisfy the triangle inequality.

To make it simple, we first define the difference between two variables from two records taking into account the normalization of the data. That is,

$$
\operatorname{diff} f_{i}(a, b)=\frac{V_{i}^{X}(a)-\overline{V_{i}^{X}}}{\sigma\left(V_{i}^{X}\right)}-\frac{V_{i}^{Y}(b)-\overline{V_{i}^{Y}}}{\sigma\left(V_{i}^{Y}\right)}
$$

In a formal way, we redefine $d^{2} E D(a, b)$ as follows:

$$
d^{2}(a, b)=\sum_{i=1}^{n} \frac{1}{n}\left(\operatorname{diff} f_{i}(a, b)\right)^{2}
$$

In addition, we will refer to each squared term of this distance as

$$
d_{i}^{2}(a, b)=\left(\operatorname{diff}_{i}(a, b)\right)^{2}
$$

\footnotetext{
${ }^{1} \mathrm{~A}$ symmetric matrix $M$ is said to be a positive definite if $x^{\prime} M x>0$ for all non zero vectors $x$, and positive semi-definite if $x^{\prime} M x \geq 0$ for all vectors $x$.
} 
Using these expressions we can define the square of the Euclidean distance as follows.

Definition 5. Given two datasets $X$ and $Y$ the square of the Euclidean distance for variable-standardized data is defined by:

$$
d^{2} A M(a, b)=A M\left(d_{1}^{2}(a, b), \ldots, d_{n}^{2}(a, b)\right),
$$

where $A M$ is the arithmetic mean $A M\left(c_{1}, \ldots, c_{n}\right)=\sum_{i} c_{i} / n$.

In general, any aggregation operator $\mathbb{C}[36]$ might be used in the place of arithmetic mean. It is important to note that not all aggregation operators will satisfy all the metric/distance properties. However, as we will show, most of the parametrized distances presented in this paper satisfy the distance properties explained in Definition 1, so we call them distances.

We can consider the following generic function.

$$
d^{2} \mathbb{C}(a, b)=\mathbb{C}\left(d_{1}^{2}(a, b), \ldots, d_{n}^{2}(a, b)\right)
$$

From this definition, it is straightforward to consider weighted versions of the $d^{2} E D(a, b)$. We briefly revise two of them below.

Definition 6. Let $p=\left(p_{1}, \ldots, p_{n}\right)$ be a weighting vector (i.e., $p_{i} \geq 0$ and $\left.\sum_{i} p_{i}=1\right)$. Then, square of the weighted mean is defined as:

$$
d^{2} W M_{p}(a, b)=W M_{p}\left(d_{1}^{2}(a, b), \ldots, d_{n}^{2}(a, b)\right),
$$

where $W M_{p}=\left(c_{1}, \ldots, c_{n}\right)=\sum_{i} p_{i} \cdot c_{i}$.

In the context of supervised learning approaches for disclosure risk evaluation this was first used in $[27,28]$. The interest of this definition is that it does not assume that all attributes are equally important in the re-identification process, since there is a weight for each attribute expressing its relevance in the re-identification process. However, it is easy to see that when null weights $\left(p_{i}=0\right)$ and the square of the function are considered, the identity of indiscernibles and the triangle inequality (Definition 2) are not satisfied.

Another type of distance is based on the Choquet integral (Definition 7, see below). This was first introduced in the context of data privacy in [29]. From a definitional point of view, its main difference with respect to the weighted distance is the use of fuzzy measures. Choquet integrals, with fuzzy measures permit us to represent, in the computation of the distance, 
information like redundancy, complementariness, and interactions among the variables, which are not used in the weighted mean. Therefore, tools that use fuzzy measures to represent background knowledge permit us to consider variables that, for example, are not independent.

Definition 7. Let $\mu$ be an unconstrained fuzzy measure on the set of variables $V$, i.e. $\mu(\emptyset)=0, \mu(V)=1$, and $\mu(A) \leq \mu(B)$ when $A \subseteq B$ for $A \subseteq V$, and $B \subseteq V$. Then, the square of the Choquet integral distance is defined as:

$$
d^{2} C I_{\mu}(a, b)=C I_{\mu}\left(d_{1}^{2}(a, b), \ldots, d_{n}^{2}(a, b)\right),
$$

where $C I_{\mu}\left(c_{1}, \ldots, c_{n}\right)=\sum_{i=1}^{n}\left(c_{s(i)}-c_{s(i-1)}\right) \mu\left(A_{s(i)}\right)$, given that $c_{s(i)}$ indicates a permutation of the indices so that $0 \leq c_{s(1)} \leq \ldots \leq c_{s(i-1)}, c_{s(0)}=0$, and $A_{s(i)}=\left\{c_{s(i)}, \ldots, c_{s(n)}\right\}$.

As in Definition 6, the Choquet integral based distance cannot be considered a metric because it does not satisfy the triangle inequality and the identity of indiscernibles properties. Nevertheless, it is shown in [37] that the Choquet integral, with respect to a submodular measure, can be used to define a metric. That is, it is possible to use the Choquet integral as a metric just adding the following condition (submodularity) to the fuzzy measure:

$$
\mu(A)+\mu(B) \geq \mu(A \cup B)+\mu(A \cap B)
$$

for all $A, B \subseteq V$.

In Section 5.2 are presented the necessary problem constraints in order to consider the weighted mean $\left(d^{2} W M\right)$ and the Choquet integral $\left(d^{2} C I\right)$ as two distance functions.

Now, we present the symmetric bilinear form. Given a vector space $V$ over a field $F$, a bilinear form is a function $B: V \times V \rightarrow F$ which satisfies the following axioms for all $w, v, u \in V$ :

1. $B(v+u, w)=B(v, w)+B(u, w)$

2. $B(w, v+u)=B(w, v)+B(w, u)$

3. $B(\alpha v, w)=B(v, \alpha w)=\alpha B(v, w)$

4. $B(v, w)=B(w, v)$

Given a square matrix $\Sigma$, we define a bilinear form for all $v, w \in V$ as $B(v, w)=v^{\prime} \Sigma w$. This form satisfies the axioms because of the distributive laws and the ability to pull out a scalar in matrix multiplication. Note 
that the matrix $\Sigma$ of a symmetric bilinear form must be itself symmetric. The symmetric bilinear functions can be considered a generalization of the Mahalanobis distance.

Then, we can use this symmetric bilinear form on the light of previous definitions as:

Definition 8. Let $\Sigma$ be $a n \times n$ symmetric weighting matrix. Then, the square of a symmetric bilinear form is defined as:

$$
d^{2} S B(a, b)=S B_{\Sigma}\left(\operatorname{dif} f_{1}(a, b), \ldots, \operatorname{dif} f_{n}(a, b)\right)
$$

where $S B_{\Sigma}\left(c_{1}, \ldots, c_{n}\right)=\left(c_{1}, \ldots, c_{n}\right)^{\prime} \Sigma\left(c_{1}, \ldots, c_{n}\right)$.

Learning the symmetric weighting matrix $\Sigma$ allows us to find which are the attributes and tuples of attributes that are more relevant in the reidentification process. That is, the diagonal expresses the relevance of each single attribute, while the upper or lower values of the weighting matrix correspond to the weights that evaluate all the interactions between each pair of attributes in the re-identification process.

If the matrix $\Sigma$ satisfies the symmetry and the positive definiteness property all the distance properties of Definition 1 are satisfied. On the contrary, if this matrix restriction is weaker, the matrix is positive semi-definite, the identity of indiscernibles is not fulfilled. Thus, there will be situations where $d(a, b)=0$ for all $a \neq b$, and then, the defined operator cannot be considered a distance anymore, it is a pseudo-distance. A clear example is when $\Sigma$ is completely null. Finally, if $\Sigma$ is neither positive definite neither positive semi-definite, i.e. negative definite, only one metric property is satisfied, the symmetry.

As we do not want negative distance values, the only requirement on $\Sigma$ we have considered is that it should be at least a positive semi-definite matrix.

Unlike the standard methods, such us the arithmetic mean (Definition 5), the interest of using Definitions 6,7 and 8 is that they give different degrees of importance to variables in the re-identification process. This would be the case if one of the variables is a key-variable, e.g. a variable where $V_{i}^{X}=V_{i}^{Y}$. In this case, all the variable weights should be zero except for the key-variable weight which should be assigned to one. Such an approach would lead to $100 \%$ of re-identifications. This is a clear example that justifies our decision to considered null weights. Taking into account null weights it 
is possible to analyze which of the variables are completely useless in the reidentification process. However, this choice forces us to renounce the identity of indiscernibles metric property.

Note that in Definition 7 and 8 the interactions of different variables are taken into account by means of the fuzzy measure and the matrix $\Sigma$ respectively. Otherwise, in Definition 6, the weighting vector can only weight the variables individually.

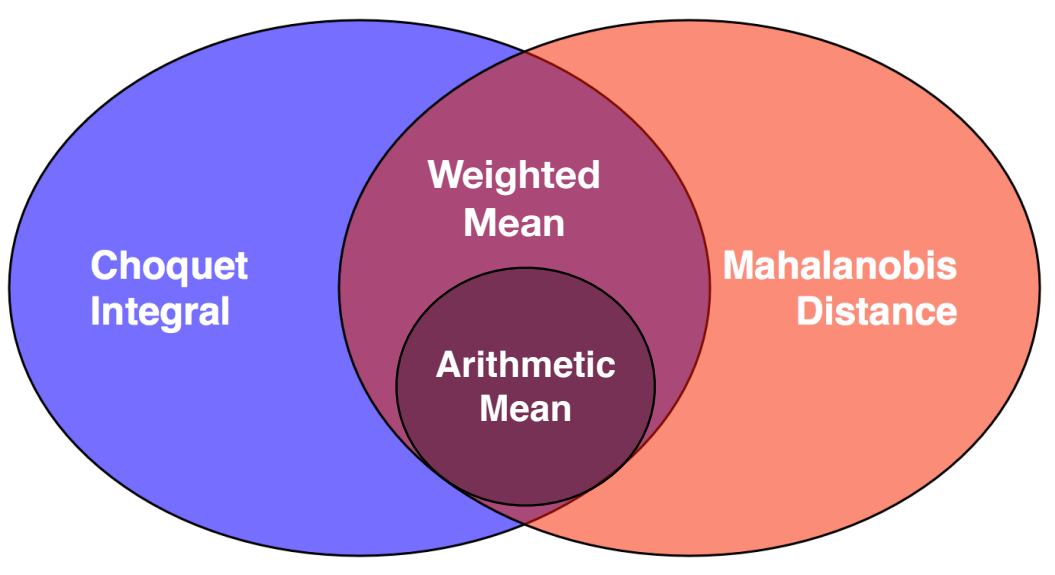

Figure 1: Distances classifications.

Figure 1 illustrates the classification of the different distances that we have explained in this section. As you can see the arithmetic mean is a special case of the weighted mean and at the same time the weighted mean is also a special case of both the Choquet integral and the Mahalanobis distance. Some more details about these relationships can be found in [38].

\section{Supervised Learning for Record Linkage}

In this section we review the general formalization of the stated optimization problem for record linkage as well as the three described aggregation operators. Section 5.1 reviews the general optimization problem which was first introduced in [28]. Afterwards, in Section 5.2 we present the formalization problems for the weighted mean and the Choquet integral operators, i.e. Definitions 6 and 7 . We also discuss the necessary problem modifications in order to satisfy all distance properties. Finally, Section 5.3 describes 
the optimization problem formalization for the proposed symmetric bilinear function.

\subsection{General Supervised Learning Approach for Record Linkage}

We describe the formalization of the general supervised metric learning problem for distance-based record linkage. This formalization is presented as a generalization of the record linkage problem independent of any parameterized function. Defining the problem in a general form allows us to create multiple variations of the problem depending on the parameterized distance function used. This problem variations rely on the specific parameterized function requirements that should be added to the problem as a set of constraints.

The problem is modeled as a Mixed Integer Linear mathematical optimization (MILP). More formally, the stated problem is expressed with a linear objective function and it is subject to a set of linear equalities and inequalities constraints. The difference between MILP and Linear Programming (LP) lies in the type of the variables considered. LP just considers real-valued variables whereas, MILP involves problems in which only some variables are constrained to be integers and the other variables are allowed to be non-integers (real). This fact makes MILPs harder problems. That is, LPs can be solved in polynomial time while, MILPs there are NP-complete problems [39] and therefore, there is no known polynomial-time algorithm.

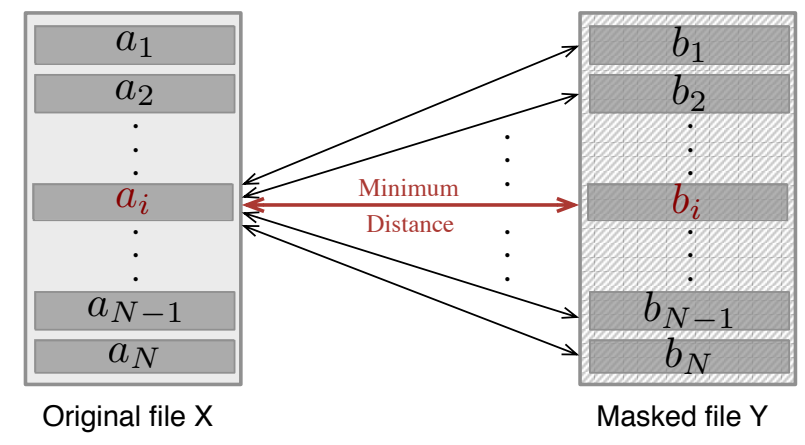

Figure 2: Distances between aligned records should be minimum.

For the sake of simplicity in the formalization of the process, we assume that each record $b_{i}$ of $Y$ is the protected version of $a_{i}$ of $X$. That is, files 


\begin{tabular}{|c|c|c|}
\hline Block & Aggregator function & Label \\
\hline \multirow{3}{*}{$K_{1}$} & $\mathbb{C}_{p}\left(a_{1}, b_{1}\right)$ & must-link \\
& $\mathbb{C}_{p}\left(a_{1}, b_{i}\right)$ & cannot-link \\
& $\mathbb{C}_{p}\left(a_{1}, b_{N}\right)$ & cannot-link \\
\hline$\vdots$ & $\vdots$ & $\vdots$ \\
\hline \multirow{2}{*}{$K_{i}$} & $\mathbb{C}_{p}\left(a_{i}, b_{1}\right)$ & cannot-link \\
& $\mathbb{C}_{p}\left(a_{i}, b_{i}\right)$ & must-link \\
& $\mathbb{C}_{p}\left(a_{i}, b_{N}\right)$ & cannot-link \\
\hline$\vdots$ & $\vdots$ & $\vdots$ \\
\hline & $\mathbb{C}_{p}\left(a_{N}, b_{1}\right)$ & cannot-link \\
$K_{N}$ & $\mathbb{C}_{p}\left(a_{N}, b_{i}\right)$ & cannot-link \\
& $\mathbb{C}_{p}\left(a_{N}, b_{N}\right)$ & must-link \\
\hline
\end{tabular}

Table 1: Data to be considered in the learning process.

are aligned. Then, two records are correctly linked using a parameterized aggregation function, $\mathbb{C}_{p}$, when the distance between the records $a_{i}$ and $b_{i}$ is smaller than the distance between the records $a_{i}$ and $b_{j}$ for all other $j$ different than $i$. So, records belonging to the same entity are considered less distant in terms of the aggregation function. Figure 2 shows an illustration of this scenario. Formally, we have that a record $a_{i}$ is correctly matched when the following equation holds for all $i \neq j$.

$$
\mathbb{C}_{p}\left(a_{i}, b_{i}\right)<\mathbb{C}_{p}\left(a_{i}, b_{j}\right)
$$

In optimal conditions these inequalities should be true for all records $a_{i}$. Nevertheless, we cannot expect this to hold because of the errors in the data caused by the protection method. Then, the learning process is formalized as an optimization problem with an objective function and some constraints.

Equation (1) should be relaxed so that the solution violates some equations. The relaxation is based on the concept of blocks. We consider a block as the set of equations concerning record $a_{i}$. Therefore, we define a block as the set of all the distances between one record of the original data and all the records of the protected data. Then, we assign to each block a variable $K_{i}$. Therefore, we have as many $K_{i}$ as the number of rows of our original file. Besides, we need for the formalization a constant $C$ that multiplies $K_{i}$ to overcome the inconsistencies and satisfy the constraint.

The rationale of this approach is as follows. The variable $K_{i}$ indicates, for 
each block, if all the corresponding constraints are accomplished $\left(K_{i}=0\right)$ or not $\left(K_{i}=1\right)$. Then, we want to minimize the number of blocks non compliant with the constraints. This way, we can find the best weights that minimize the number of violations, or in other words, we can find the weights that maximize the number of re-identifications between the original and protected data. Table 1 shows a graphical example of the problem division and the information needed for the learning process, i.e., the labels of the correct links, the ones that correspond to the same individuals.

The rationale of our formalization is that if for a record $a_{i}$, Equation (1) is violated for a certain record $b_{j}$, then, it does not matter that other records $b_{h}$, where $h \neq j \neq i$, also violate the same equation for the same record $a_{i}$. This is so because record $a_{i}$ will not be re-identified.

Using these variables $K_{i}$ and the constant $C$, we have that all pairs $i \neq j$ should satisfy

$$
\mathbb{C}_{p}\left(a_{i}, b_{j}\right)-\mathbb{C}_{p}\left(a_{i}, b_{i}\right)+C K_{i}>0 .
$$

As $K_{i}$ is only 0 or 1 , we use the constant $C$ as the factor needed to really overcome the constraint. In fact, the constant $C$ expresses the minimum distance we require between the correct link and the other incorrect links. The larger it is, the more correct links are distinguished from incorrect links.

Using these constraints we can formalize the optimization problem that finds the set of parameter values defined for a given aggregation operator $\mathbb{C}$ that minimizes the number of incorrect links. That is,

$$
\text { Minimize } \sum_{i=1}^{N} K_{i}
$$

Subject to :

$$
\begin{aligned}
& \mathbb{C}_{p}\left(a_{i}, b_{j}\right)-\mathbb{C}_{p}\left(a_{i}, b_{i}\right)+C K_{i}>0, \quad \forall i, j=1, \ldots, N, i \neq j \\
& K_{i} \in\{0,1\}
\end{aligned}
$$

This is an optimization problem with a linear objective function and linear constraints (Equations (3) and (4)). However, depending on which aggregation operator $\mathbb{C}_{p}$ we decide to use, we will have to add some additional constraints related to that aggregation operator and its parameters. In addition, we have to pay special attention to which is the polynomial degree of the aggregation operator we want to use and the parameter constraints, because it could lead us to deal with non-linear or non-quadratic programming problem. 
If $N$ is the number of records, and $n$ the number of variables of the two data sets $X$ and $Y$. Then, the objective function, Equation (2), consists of a summation of $N$ control variables, one per each defined distances' block, i.e., $K_{i}$ for all $i=1 \ldots N$. With respect to the total number of problem constraints; there are $(N(N-1))$ constraints concerning to Equation (3) and $N$ constraints defining the control variable, Equation (4). Therefore, there are a total of $(N(N-1))+N$ constraints. Note that depending on the aggregation function $\mathbb{C}_{p}$ used, there will be more constraints in the problem. We will discuss the number of such constraints in the particular problems below.

\subsection{Learning the Optimal Weights Using $d^{2} W M$ an $d^{2} C I$}

We outline in Table 2 the necessary extra constraints to formalize the general optimization problem (Equations (2), (3) and (4)) for the weighted mean and the Choquet integral operators (Definitions 6 and 7 respectively). The table also includes the number of constraints of the optimization problem in each case. More details and deeper explanations can be found in the following works $[28,29]$.

\begin{tabular}{l|l|l}
\hline & $d^{2} W M$ & $d^{2} C I$ \\
\hline Additional & $\sum_{i=1}^{n} p_{i}=1$ & $\mu(\emptyset)=0$ \\
Constraints & $p_{i} \geq 0$ & $\mu(V)=1$ \\
& & $\mu(A) \leq \mu(B)$ when $A \subseteq B$ \\
\hline Total Constr. & $N(N-1)+N+n+1$ & $N(N-1)+N+2+\sum_{k=2}^{n}\left(\begin{array}{l}n \\
k\end{array}\right) k$ \\
\hline
\end{tabular}

Table 2: Additional constraints of the weighted mean and Choquet integral.

As was mention in Section 4 none of the aggregators showed in Table 2 satisfy completely the distance properties. Therefore, following the instructions given in that section we show in Table 3 which are the set of corresponding changes that have to be applied to each optimization problem. Thus, both the weighted mean and the Choquet integral can be considered distance functions.

Note that in all cases the additional constraints are linear. They are mixed integer linear problems (MILP), because they are dealing with integers and real-valued. Note, that we only have considered aggregation operators with real-valued weights. 


\begin{tabular}{l|l|l}
\hline & $d^{2} W M_{m}$ & $d^{2} C I_{m}$ \\
\hline Additional & $\sum_{i=1}^{n} p_{i}=1$ & $\mu(\emptyset)=0$ \\
Constraints & $p_{i}>0$ & $\mu(V)=1$ \\
& & $\mu(A) \leq \mu(B)$ when $A \subseteq B$ \\
& & $\mu(A)+\mu(B) \geq \mu(A \cup B)+\mu(A \cap B)$ \\
\hline Total Constr. & $N(N-1)+N+1+n$ & $N(N-1)+N+2+\left(\sum_{k=2}^{n}\left(\begin{array}{l}n \\
k\end{array}\right) k\right)+\left(\begin{array}{l}n \\
2\end{array}\right)$ \\
\hline
\end{tabular}

Table 3: Additional constraints of the weighted mean and Choquet integral as distances.

\subsection{Learning the Optimal Weights Using a Symmetric Bilinear Form}

In this section we define the optimization problem and the specific constraints when $\mathbb{C}$ is based on Definition 8 (a symmetric bilinear function). The minimization problem is expressed as:

Minimize $\sum_{i=1}^{N} K_{i}$

Subject to :

$d^{2} S B_{\Sigma}\left(a_{i}, b_{j}\right)-d^{2} S B_{\Sigma}\left(a_{i}, b_{i}\right)+C K_{i}>0, \quad \forall i, j=1, \ldots, N, i \neq j$

$\Sigma \succeq 0$

$K_{i} \in\{0,1\}$

where, as before, $N$ is the number of records, and $n$ the number of attributes of the input files.

One of the required distance properties for the matrix $\Sigma$ in Definition 8 was its positive semi-definiteness. To ensure this property, we can solve the problem with Semi-definite programming (SDP) or using other methods that ensure the symmetry of the matrix and also that the matrix has non-negative eigenvalues [40]. Nevertheless, none of these approaches are technically feasible with linear constraints (they can only be formalized with non-linear constraints). To avoid the non-linear constrains we have considered two approximations.

The first approximation $\left(d^{2} S B\right)$ consists in changing Equation $(7)$ of the previous formalization by the following linear constraint:

$$
d^{2} S B_{\Sigma}\left(a_{i}, b_{j}\right) \geq 0, \quad \forall i, j=1, \ldots, N
$$

Equation (9) forces the distance to be semi-positive for all pairs of records $\left(a_{i}, b_{j}\right)$ in the input set. Although, the $\Sigma$ positive semi-definite is not ensured, 
this approximation ensures that non-negativity will be satisfied for the input dataset.

The second approximation $\left(d^{2} S B_{N C}\right)$ does not consider the matrix restriction in the formalization of the optimization problem. Thus, the problem consists of a linear objective function Equation (5) and two linear constraints Equations (6) and (8). Thus, this approach consists of solving the stated optimization problem and then, do a post-processing of the resulting matrix $\Sigma$. We apply the Higham's algorithm [26] to the matrix $\Sigma$. This method computes the nearest positive semi-definite matrix from a non-positive definite.

Both proposed approximations have to determine the same number of parameters, $n(n+1) / 2$. They correspond to the diagonal and the upper (or lower) triangle of the matrix $\Sigma$. The first approach consists of a linear objective function plus $N(N-1)+N+N^{2}$ constraints. That is, the general plus all constraints related to Equation (9). While, the second approach considers the same number of constraints as the general optimization problem: $N(N-1)+N$.

\section{Evaluation}

Given an original file and its masked version, we pre-process and build the problem structure by means of a series of $\mathrm{R}$ functions, then following this formalized structure the problem is expressed into MPS (Mathematical Programming System) file format. MPS is a file format to represent and store Linear Programming (LP) and Mixed Integer Programming (MIP) problems. Then, each file is processed with an optimization solver. We solve our experiments with one of the most used commercial solvers, the IBM ILOG CPLEX tool [41] (version 12.1). Thus, for each formalized problem this solver finds

the corresponding parameter values that maximize the number of correct links between the original and the masked data.

The experiments were performed in the Finis Terrae computer from the supercomputing center of Galicia [42]. Finis Terrae is composed of $142 \mathrm{HP}$ Integrity rx7640 computing nodes with 16 Itanium Montvale cores and 128 GB of memory each, one HP Integrity Superdome node, with 128 Itanium Montvale cores and 1,024GB of memory, and 1 HP Integrity Superdome node, with 128 Itanium 2 cores and $384 \mathrm{~GB}$ of memory. From the Finis Terrae computer we used 16 cores and $32 \mathrm{~GB}$ of ram memory. 


\subsection{Test Set}

A data file was protected by a perturbative approach called microaggregation [9], a well-known microdata protection method, which broadly speaking, provides privacy by means of clustering the data into small clusters of size at least $k$, and then replacing the original data by the centroid of their corresponding clusters. This parameter $k$ determines the protection level: the greater the $k$, the greater the protection and at the same time the greater the information loss.

We have considered files with the following protection parameters:

- M4-33: 4 variables microaggregated in groups of 2 with $k=3$.

- M4-28: 4 variables, first 2 variables with $k=2$, and last 2 with $k=8$.

- M4-82: 4 variables, first 2 variables with $k=8$, and last 2 with $k=2$.

- M5-38: 5 variables, first 3 variables with $k=3$, and last 2 with $k=8$.

- M6-385: 6 variables, first 2 variables with $k=3$, next 2 variables with $k=8$, and last 2 with $k=5$.

- M6-853: 6 variables, first 2 variables with $k=8$, next 2 variables with $k=5$, and last 2 with $k=3$.

For each case, we have protected 400 records randomly selected from the Census dataset [43] from the European CASC project [44], which contains 1080 records and 13 variables, and has been extensively used in other works $[45,46,47]$.

\begin{tabular}{|c|r|r|}
\hline Attr. & Mean & Std dev $(\sigma)$ \\
\hline$V_{1}$ & $196,039.8$ & $101,251.417$ \\
$V_{2}$ & $56,222.76$ & $24,674.843$ \\
$V_{3}$ & $3,173.135$ & $1,401.832$ \\
$V_{4}$ & $7,544.656$ & $4,905.200$ \\
$V_{5}$ & $45,230.84$ & $21,323.470$ \\
$V_{6}$ & $2,597.184$ & $1,826.436$ \\
\hline
\end{tabular}

Table 4: Mean and standard deviation $(\sigma)$ for each column attribute.

In Table 4 we provide some basic statistical information from the Census dataset, such as the mean and the standard deviation for the first six columns. 
From it we can see how different are the data attributes in terms of their means, and also how spread out are the data points over a large range of values. In addition, in Figure 3 is shown a graphical representation of the Pearson correlation coefficient, which indicates a degree of linear relationship between all pairs of attributes.

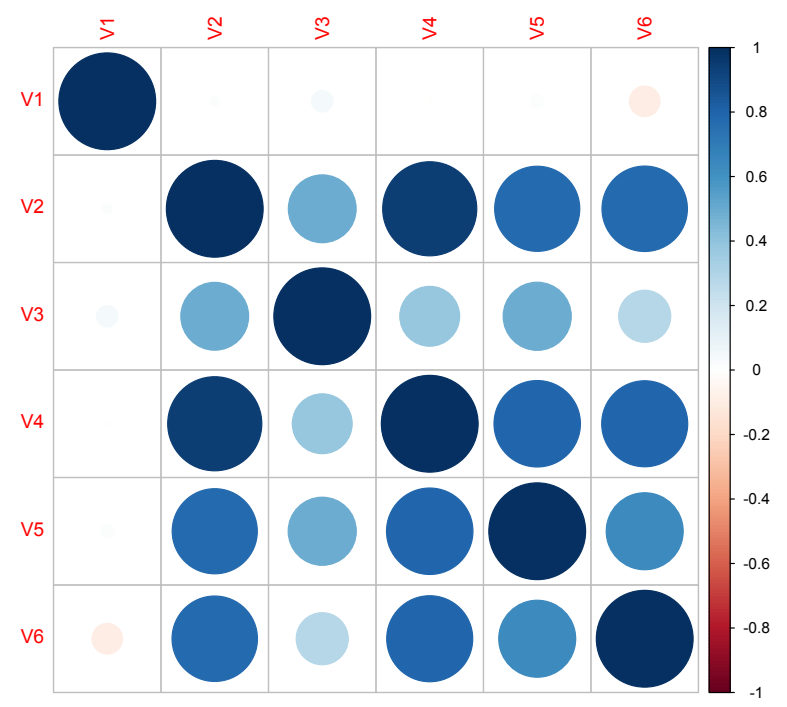

Figure 3: Graphical representation of the Census data set correlation matrix.

Note that in our experiments we apply different protection degrees to different variables of the same file. The values used range between 2 to 8 , i.e., values between the lowest protection value and a good protection degree in accordance to [18]. This is especially interesting when variables have different sensitivity. We have used the web application [48], which is based on [18], to compute standard scores to evaluate all the protected datasets. These scores are computed by means of a combination of information loss and disclosure risk values, so the best protection method is the one that optimizes the trade-off between the information loss and the disclosure risk. Table 5 shows the average record linkage, the probabilistic information loss and the overall score for all the protected files. The best score is achieved by the M5-38 file, though the other files have a very similar score. 


\begin{tabular}{|l|l|l|l|}
\hline & AvRL(\%) & PIL $(\%)$ & Score $(\%)$ \\
\hline M4-33 & 42.127 & 23.85 & 32.99 \\
M4-28 & 33.47 & 28.40 & 30.94 \\
M4-82 & 32.37 & 31.80 & 32.09 \\
M5-38 & 26.01 & 31.92 & $\mathbf{2 8 . 9 6}$ \\
M6-385 & 35.42 & 36.91 & 36.16 \\
M6-853 & 30.65 & 37.76 & 34.21 \\
\hline
\end{tabular}

Table 5: Evaluation of the protected datasets.

\subsection{Results}

Table 6 shows the linkage percentage using different approaches for record linkage. These percentages determine the maximum number of correctly identified records from the total, so a value of 100 means that all records from the original and the masked data were correctly linked (re-identified). The maximum number of correctly linked records are determined by the CPLEX solver for each generated MILP problem.

The approaches considered are the following ones: the standard record linkage method $\left(d^{2} A M\right)$; the Mahalanobis distance $\left(d^{2} M D\right)$; two supervised learning approaches: the weighted mean $\left(d^{2} W M\right)$ and the Choquet integral $\left(d^{2} C I\right)$ and their corresponding proposed versions satisfying the distance properties $\left(d^{2} W M_{m}\right.$ and $\left.d^{2} C I_{m}\right)$, which were described in Section 4; and finally, the new supervised learning approaches, which are based on a symmetric bilinear form $\left(d^{2} S B\right.$ and $\left.d^{2} S B_{N C}\right)$. Recall that whereas $d^{2} S B_{N C}$ is the approach formed by Equations (5), (6) an (8), $d^{2} S B$ has an extra constraint, Equation (9).

Recall that due to the lack of constraints in the $d^{2} S B_{N C}$ problem formalization, it is possible the solver finds a matrix that does not satisfy the positive semi-definiteness property. In these particular problems, we have applied the Higham's algorithm [26] to the resulting matrix $\Sigma$. Thus, we are able to compute its nearest positive semi-definite matrix. Then, we check manually the number of correctly linked records with the symmetric bilinear function (Definition 8) and the matrix computed by the Higham's algorithm. These cases are named $d^{2} S B_{P D}$.

Before tackling the results obtained by the presented supervised approaches we focus on the non-supervised approaches. The most noticeable fact between the standard distance-based record linkage $\left(d^{2} A M\right)$ and Mahalanobis distance $\left(d^{2} M D\right)$ is the improvement achieved by the latter method, which 


\begin{tabular}{lrrrrrr} 
& $M 4-33$ & $M 4-28$ & $M_{4-82}$ & $M 5-38$ & $M 6-385$ & $M 6-853$ \\
\hline$d^{2} A M$ & 84.00 & 68.50 & 71.00 & 39.75 & 78.00 & 84.75 \\
$d^{2} M D$ & 94.00 & 90.00 & 92.75 & 88.25 & 98.50 & 98.00 \\
\hline$d^{2} W M$ & 95.50 & 93.00 & 94.25 & 90.50 & 99.25 & 98.75 \\
$d^{2} W M_{m}$ & 95.50 & 93.00 & 94.25 & 90.50 & 99.25 & 98.75 \\
$d^{2} C I$ & 95.75 & 93.75 & 94.25 & 91.25 & $\mathbf{9 9 . 7 5}$ & 99.25 \\
$d^{2} C I_{m}$ & 95.75 & 93.75 & 94.25 & 90.50 & 99.50 & 98.75 \\
$d^{2} S B_{N C}$ & $\mathbf{9 6 . 7 5}$ & $\mathbf{9 4 . 5}$ & $\mathbf{9 5 . 2 5}$ & $\mathbf{9 2 . 2 5}$ & $\mathbf{9 9 . 7 5}$ & $\mathbf{9 9 . 5 0}$ \\
$d^{2} S B$ & $\mathbf{9 6 . 7 5}$ & $\mathbf{9 4 . 5}$ & $\mathbf{9 5 . 2 5}$ & $\mathbf{9 2 . 2 5}$ & $\mathbf{9 9 . 7 5}$ & $\mathbf{9 9 . 5 0}$ \\
$d^{2} S B_{P D}$ & - & - & - & - & - & 99.25 \\
\hline
\end{tabular}

Table 6: Percentage of the number of correct re-identifications.

in average achieves about $22.6 \%$ more correct re-identifications and for the protected file $M 5-38$ achieves a maximum improvement of $48.5 \%$. This improvement and ease computation of Mahalanobis distance makes that $d^{2} M D$ should be strongly considered for the disclosure risk assessment of protected datasets. However, as it is also shown in Table 6, these results can still be overcome by the presented optimization approaches.

We first compare the presented symmetric bilinear function approaches $\left(d^{2} S B\right.$ and $\left.d^{2} S B_{N C}\right)$ with the weighted mean $\left(d^{2} W M\right)$ and the Choquet integral $\left(d^{2} C I\right)$ approaches. The results obtained by these supervised approaches show that almost for all the protected files the optimization problem with respect to the symmetric bilinear function $\left(d^{2} S B\right.$ and $\left.d^{2} S B_{N C}\right)$ achieves the larger number of correct matches. Their results are slightly followed by the Choquet integral $\left(d^{2} C I\right)$ by a maximum difference of exactly $1 \%$ (4 correct matches less) for Mic3-44 and Mic5-38 protections. Improvements obtained by the Choquet integral are also slightly followed by the ones obtained by the weighted mean approach $\left(d^{2} W M\right)$, which has a maximum difference of $0.75 \%$ (3 correct matches less) for Mic4-28 protection. In terms of accuracy (number of records correctly re-identified) we can conclude that from the supervised learning approaches the symmetric bilinear, the Choquet integral and the weighted mean are the best methods. Then, we compare their accuracies in those protected files where the standard record linkage approach $\left(d^{2} A M\right)$ achieve the maximum (M6-853) and the minimum (Mic5-38) number of re-identifications. We obtained an improvement of $14.76 \%$ (by $d^{2} S B$ ), $14.51 \%$ (by $d^{2} C I$ ) and $14.01 \%$ (by $d^{2} W M$ ) for the $M 6-853$ file and improvement of $52.5 \%$ (by $d^{2} S B$ ), $51.5 \%$ (by $d^{2} C I$ ) and $50.751 \%$ (by $d^{2} W M$ ) for 
the M5-38 file. However, to evaluate all approaches it is also important to bear in mind the problem complexity and its computing time, factor that we analyze below, in Table 7 .

We now focus on the results obtained by the weighted mean $\left(d^{2} W M\right)$, the Choquet integral $\left(d^{2} C I\right)$ and their respectively modified versions which satisfy all or almost all the metric properties $\left(d^{2} W M_{m}\right.$ and $\left.d^{2} C I_{m}\right)$. Table 6 shows how similar they are. Comparing the $d^{2} W M$ and $d^{2} W M_{m}$ reidentification percentages we appreciate that both approaches obtain exactly the same values. With respect to the Choquet integral approaches we see the not modified version $\left(d^{2} C I\right)$ achieves slightly better results in some of the datasets tested than $d^{2} C I_{m}$. Therefore, despite of adding new constraints to the problem there is a slight decrease (or none decrease) in the number of re-identifications.

Finally, we focus on both symmetric bilinear approximations. Let us underline that all matrices by $d^{2} S B$ and $d^{2} S B_{N C}$ satisfy the positive definiteness property, except for the last dataset (M6-853), which in either of the two approaches this property was not satisfied. The Higham's algorithm was applied to the matrix obtained by the solver for the $d^{2} S B_{N C}$ approach achieving a new positive definite matrix. $d^{2} S B_{P D}$ in Table 6 shows the percentage results for this test case. We note that the percentage of correct re-identifications is slightly lower than for $d^{2} S B_{N C}$ but is still higher than the rest of the analyzed methods. Recall that when the obtained matrix is positive definite all distance properties are satisfied as well as the identity of indiscernibles. Using the symmetric bilinear approach with a positive semidefinite matrix achieves better results that the Mahalanobis distance using the covariance matrix compute from the data.

\begin{tabular}{lrrrrrr} 
& M4-33 & M4-28 & M4-82 & M5-38 & M6-385 & M6-853 \\
\hline$d^{2} W M$ & 29.83 & 41.37 & 24.33 & 718.43 & 11.81 & 17.77 \\
$d^{2} W M_{m}$ & 3.43 & 6.26 & 2.26 & 190.75 & 4.34 & 6.72 \\
$d^{2} C I$ & 280.24 & 427.75 & 242.86 & $42,731.22$ & 24.17 & 87.43 \\
$d^{2} C I_{m}$ & 155.07 & 441.99 & 294.98 & $4,017.16$ & 79.43 & 829.81 \\
$d^{2} S B_{N C}$ & 32.04 & $2,793.81$ & 150.66 & $10,592.99$ & 13.65 & 14.11 \\
$d^{2} S B$ & 13.67 & $3,479.06$ & 139.59 & $169,049.55$ & 13.93 & 13.70 \\
\hline
\end{tabular}

Table 7: Computation time comparison (in seconds).

The computation time taken to learn the optimal weights for each dataset and learning approach can be seen below, in Table 7 . 
Moreover, we have compared the covariance matrices used in $d^{2} M D$ and the inverses of the weighting matrices obtained by the supervised approach using the symmetric bilinear function $d^{2} S B_{N C}$ for the first five datasets and the matrix obtained by $d^{2} S B_{P D}$ for the last case (because of the positive semidefiniteness. These are supposed to be similar than the covariance matrices or a scaled variation of those. However, when we compare both matrices by means of the mean square error (Equation 10), the results show that both matrices are different. See Table 8.

$$
\operatorname{MSE}\left(V, V^{\prime}\right)=\frac{\sum_{j=1}^{n} \sum_{1 \leq i \leq j}\left(v_{i j}-v_{i j}^{\prime}\right)^{2}}{\frac{n(n+1)}{2}}
$$

\begin{tabular}{|l|l|}
\hline & Mean square error \\
\hline M4-33 & 18.49 \\
M4-28 & 48.75 \\
M4-82 & $2,784.81$ \\
$M 5-38$ & 7.26 \\
$M 6-385$ & $15.91 \times 10^{6}$ \\
$M 6-853$ & $12.77 \times 10^{16}$ \\
\hline
\end{tabular}

Table 8: Mean square error between covariance matrices and the positive definite matrices obtained.

\section{Conclusions}

In this paper we introduced a new supervised learning approach and a parameterized aggregator, a symmetric bilinear function, to solve record linkage problems. This approach is formalized as an optimization problem defined by a set of cannot-link and must-link constraints. Thus, the problem is solved by finding the parameter values of the symmetric bilinear function that maximizes the number of correct links between two datasets. We have compared this supervised learning method with other supervised and non-supervised ones.

Our experiments have been done in the area of data privacy. In this area, record linkage is used to evaluate disclosure risk. It is used to link records of the original and the protected file, modeling the attack of an intruder that wants to disclose information from the protected file. We have focused on 
the worst case. This is the case in which the person who wants to do the re-identification has the entire original database. Note that as the original data is confidential this scenario is only applicable by the data owner to evaluate the risk of the protected file. The parameterized distance based record linkage is a very useful tool for the data owner, not only to evaluate the disclosure risk of the protected database before its release, but also to know which are the variables or sets of variables that maximize the number of re-identifications and make weaker the protected data. This estimation is based on the number of correct links between the original and the protected data.

The experiments show that the proposed approach is the one that achieves the best results. Although, the improvement is not very high, especially when we compare it with the other parametrized variations, it is relevant for the evaluation of risk of a protected dataset. Moreover, by means of analyzing the weights obtained it is possible to identify the variables and sets of variables that clearly provide more information for an attacker of the database. This is useful in the data protection process. For instance, when a variable provides more information than the others, we would apply a higher degree of protection or even another protection method to make it more secure.

In this paper we also introduced two variations for the weighted mean and Choquet problems in order to be considered metrics $\left(d^{2} W M_{m}, d^{2} C I_{m}\right)$. We can conclude that for our problem, the record linkage for disclosure risk evaluation, they are not promising, so the number of re-identifications slightly decrease when are compared with their original approaches $\left(d^{2} W M, d^{2} C I\right)$. Besides, in the case of $d^{2} W M_{m}$ null weights are not considered and so there is a lack of information for those variable which are not relevant in the reidentification process.

As future work we consider developing optimization problems that are non-linear programming ones. For example, to consider the case in which the weighting matrix satisfies the positive semi-definiteness property of the covariance matrix. We need them to compare the results and computational time of such approach with the other presented methods. Furthermore, it would be interesting a comparison between the described supervised learning approach relying on the Choquet integral and a similar semi-supervised metric learning research approach proposed by Beliakov et al. in [23]. 


\section{Acknowledgments}

Partial supports by the Spanish MICINN (projects ARES-CONSOLIDER INGENIO 2010 CSD2007-00004, TIN2010-15764 and TIN2011-27076-C0303) and by the EC (FP7/2007-2013) Data without Boundaries (grant agreement number 262608) are acknowledged. Some results described in this paper have been obtained using the Centro de Supercomputación de Galicia (CESGA). This partial support is gratefully acknowledged. The work contributed by the first author was carried out as part of the Computer Science Ph.D. program of the Universitat Autònoma de Barcelona (UAB).

[1] H. Dunn, Record linkage, American Journal of Public Health 36 (12) (1946) 1412-1416.

[2] H. B. Newcombe, J. M. Kennedy, S. J. Axford, A. P. James, Automatic linkage of vital records., Science 130 (1959) 954-959.

[3] H. B. Newcombe, J. M. Kennedy, Record linkage: making maximum use of the discriminating power of identifying information, Commun. ACM 5 (11) (1962) 563-566.

[4] I. Fellegi, A. Sunter, A theory for record linkage, Journal of the American Statistical Association 64 (328) (1969) 1183-1210.

[5] A. Mccallum, B. Wellner, Object consolodation by graph partitioning with a conditionally-trained distance metric, In Proceedings of the KDD2003 Workshop on Data Cleaning, Record Linkage, and Object Consolidation (2003) 19-24.

[6] W. E. Winkler, Data cleaning methods, in: Ninth ACM SIGKDD International Conference on Knowledge Discovery and Data Mining, 2003.

[7] C. Batini, M. Scannapieco, Data Quality: Concepts, Methodologies and Techniques (Data-Centric Systems and Applications), Springer-Verlag New York, Inc., 2006.

[8] A. Elmagarmid, P. G. Ipeirotis, V. S. Verykios, Duplicate record detection: A survey, IEEE Transactions on Knowledge and Data Engineering 19 (1) (2007) 1-16. 
[9] D. Defays, P. Nanopoulos, Panels of enterprises and confidentiality: The small aggregates method, in: Proc. of the 1992 Symposium on Design and Analysis of Longitudinal Surveys, Statistics Canada, 1993, pp. 195204.

[10] Statistics Canada. Record linkage at statistics canada, http://www . statcan.gc.ca/record-enregistrement/index-eng.htm (2010).

[11] data.gov.uk, Uk government (2010).

[12] data.gov, Usa government (2010).

[13] R. Agrawal, R. Srikant, Privacy-preserving data mining, in: Proc. of the ACM SIGMOD Conference on Management of Data, ACM Press, 2000, pp. 439-450.

[14] L. Willenborg, T. Waal, Elements of Statistical Disclosure Control, Springer-Verlag, 2001.

[15] W. E. Winkler, Re-identification methods for masked microdata, in: J. Domingo-Ferrer, V. Torra (Eds.), Privacy in Statistical Databases, Vol. 3050 of Lecture Notes in Computer Science, Springer Berlin Heidelberg, 2004, pp. 216-230.

[16] V. Torra, J. Abowd, J. Domingo-Ferrer, Using mahalanobis distancebased record linkage for disclosure risk assessment, in: J. DomingoFerrer, L. Franconi (Eds.), Privacy in Statistical Databases, Vol. 4302 of Lecture Notes in Computer Science, Springer Berlin Heidelberg, 2006, pp. 233-242.

[17] N. L. Spruill, Measures of confidentiality, in: Statistic of Income and Related Administrative Record Research, 1982, pp. 131-136.

[18] J. Domingo-Ferrer, V. Torra, A quantitative comparison of disclosure control methods for microdata, in: P. Doyle, J. Lane, J. Theeuwes, L. Zayatz (Eds.), Confidentiality, disclosure, and data access : Theory and practical applications for statistical agencies, Elsevier, 2001, pp. 111-133.

[19] L. Yang, R. Jin, Distance metric learning: A comprehensive survey, Tech. rep., Michigan State Universiy (2006). 
[20] E. P. Xing, A. Y. Ng, M. I. Jordan, S. Russell, Distance metric learning with application to clustering with side-information, Advances in neural information processing systems (2003) 521-528.

[21] M. Schultz, T. Joachims, Learning a distance metric from relative comparisons, in: Advances in Neural Information Processing Systems 16, MIT Press, 2004, pp. 41-48.

[22] M. Halkidi, D. Gunopulos, M. Vazirgiannis, N. Kumar, C. Domeniconi, A clustering framework based on subjective and objective validity criteria, ACM Trans. Knowl. Discov. Data 1 (4) (2008) 4:1-4:25.

[23] G. Beliakov, S. James, G. Li, Learning choquet-integral-based metrics for semisupervised clustering, Fuzzy Systems, IEEE Transactions on 19 (3) (2011) 562-574.

[24] K. Q. Weinberger, J. Blitzer, L. K. Saul, Distance metric learning for large margin nearest neighbor classification, in: Y. Weiss, B. Schölkopf, J. Platt (Eds.), Advances in Neural Information Processing Systems 18, MIT Press, 2006, pp. 1473-1480.

[25] S. Sun, Q. Chen, Hierarchical distance metric learning for large margin nearest neighbor classification, International Journal of Pattern Recognition and Artificial Intelligence 25 (07) (2011) 1073-1087.

[26] N. Higham, Computing the nearest correlation matrix a problem from finance, IMA Journal of Numerical Analysis 22 (3) (2002) 329-343.

[27] V. Torra, G. Navarro-Arribas, D. Abril, Supervised learning for record linkage through weighted means and owa operators, Control and Cybernetics 39 (4) (2010) 1011-1026.

[28] D. Abril, G. Navarro-Arribas, V. Torra, Improving record linkage with supervised learning for disclosure risk assessment, Information Fusion 13 (4) (2012) 274-284.

[29] D. Abril, G. Navarro-Arribas, V. Torra, Choquet integral for record linkage, Annals of Operations Research 195 (1) (2012) 97-110.

[30] D. Pagliuca, G. Seri, Some results of individual ranking method on the system of enterprise acounts annual survey, Esprit SDC Project, Delivrable MI-3/D2 (1999). 
[31] H. O. Hartley, Maximum likelihood estimation from incomplete data, Biometrics 14 (2) (1958) 174-194.

[32] G. McLachlan, T. Krishnan, The EM Algorithm and Extensions (Wiley Series in Probability and Statistics), Wiley-Interscience, 1997.

[33] M. A. Jaro, Advances in record-linkage methodology as applied to matching the 1985 census of tampa, florida, Journal of the American Statistical Association 84 (406) (1989) 414-420.

[34] M. Deza, E. Deza, Encyclopedia of distances, Springer Verlag, 2009.

[35] P. C. Mahalanobis, On the generalised distance in statistics, in: Proceedings National Institute of Science, India, Vol. 2, 1936, pp. 49-55. URL \url\{http://ir.isical.ac.in/dspace/handle/1/1268\}

[36] V. Torra, Y. Narukawa, Modeling Decisions: Information Fusion and Aggregation Operators, Springer, 2007.

[37] Y. Narukawa, Distances defined by choquet integral, in: Fuzzy Systems Conference, 2007. FUZZ-IEEE 2007. IEEE International, IEEE, 2007, pp. 1-6.

[38] V. Torra, Y. Narukawa, On a comparison between mahalanobis distance and choquet integral: The choquetmahalanobis operator, Information Sciences 190 (0) (2012) 56 - 63.

[39] A. Schrijver, Theory of Linear and Integer Programming, John Wiley \& Sons, Inc., New York, NY, USA, 1986.

[40] C. R. Johnson, Positive definite matrices, The American Mathematical Monthly 77 (3) (1970) pp. 259-264.

[41] I. IBM ILOG CPLEX, High-performance mathematical programming engine. international business machines corp., http://www01.ibm.com/software/integration/optimization/cplex/ (2010).

[42] CESGA, Centro de supercomputación de galicia.

URL \url\{http://www.cesga.es\}

[43] U. Census Bureau, Data extraction system, http://www.census.gov/ (1995). 
[44] R. Brand, J. Domingo-Ferrer, J. Mateo-Sanz, Reference datasets to test and compare sdc methods for protection of numerical microdata, Technical report, European Project IST-2000-25069 CASC (2002).

[45] M. Laszlo, S. Mukherjee, Minimum spanning tree partitioning algorithm for microaggregation, IEEE Trans. on Knowl. and Data Eng. 17 (7) (2005) 902-911.

[46] J. Domingo-Ferrer, V. Torra, Ordinal, continous and heterogeneous anonymity through microaggregation, Data Mining and Knowledge Discovery. 11 (2) (2005) $195-212$.

[47] W. E. Yancey, W. E. Winkler, R. H. Creecy, Disclosure risk assessment in perturbative microdata protection, in: Inference Control in Statistical Databases, From Theory to Practice, Vol. 2316, Springer-Verlag, London, UK, 2002, pp. 135-152.

[48] ppdm.iiia.csic.es, (PPDM) Privacy Preserving Data Mining (2009). 